\title{
SYNCHRONIZATION OF FRACTIONAL-ORDER DISCRETE-TIME CHAOTIC SYSTEMS BY AN EXACT DELAYED STATE RECONSTRUCTOR: APPLICATION TO SECURE COMMUNICATION
}

\author{
SAID DJENNOUNE $^{a, *}$, MAAMAR BETTAYEB $^{b, c}$, UbAid MUHSEN AL-SAGGAF $^{d}$ \\ ${ }^{a}$ Laboratory of Design and Conduct of Production Systems \\ Mouloud Mammeri University, BP 17 RP 15000, Tizi-Ouzou, Algeria \\ e-mail: s_djennoune@yahoo.fr \\ ${ }^{b}$ Department of Electrical and Computer Engineering \\ University of Sharjah, PO Box 27272, Sharjah, United Arab Emirates \\ e-mail: maamar@sharjah.ac.ae \\ ${ }^{c}$ Center of Excellence in Intelligent Engineering Systems (CEIES) \\ King Abdulaziz University, Al Ehtifalat St, 21589, Jeddah, Saudi Arabia \\ ${ }^{d}$ Electrical and Computer Engineering Department \\ King Abdulaziz University, Al Ehtifalat St, 21589, Jeddah, Saudi Arabia \\ e-mail: usaggaf@kau.edu.sa
}

\begin{abstract}
This paper deals with the synchronization of fractional-order chaotic discrete-time systems. First, some new concepts regarding the output-memory observability of non-linear fractional-order discrete-time systems are developed. A rank criterion for output-memory observability is derived. Second, a dead-beat observer which recovers exactly the true state system from the knowledge of a finite number of delayed inputs and delayed outputs is proposed. The case of the presence of an unknown input is also studied. Third, secure data communication based on a generalized fractional-order Hénon map is proposed. Numerical simulations and application to secure speech communication are presented to show the efficiency of the proposed approach.
\end{abstract}

Keywords: fractional-order discrete time systems, chaotic map, chaotic synchronization, dead-beat observer, secure data communication.

\section{Introduction}

Nowadays, it is well recognized that the concept of fractional calculus has become very popular because of its many applications in the modeling of physical phenomena, system theory and controller design (Monje et al., 2010; Sabatier et al., 2008; Magin, 2004). Fractional-order integration and differentiation operators in the field of continuous-time systems have been mostly successfully used. However, since the work of Miller and Ross (1989), we have witnessed the emergence of fractional-order difference operators in the domain of discrete-time systems. This emergence is demonstrated by

${ }^{*}$ Corresponding author the multitude of works published in the last two decades dealing with the definitions of fractional-order differences operators (Atici and Eloe, 2007; 2009; Abdeljawad and Baleanu, 2009; Holm, 2011; Ortigueira, 2000; Chen et al., 2011; Mozyrska and Pawłuszewicz, 2011), as well as Lyapunov stability (Wyrwas et al., 2015; Guermah et al., 2008a), controllability and observability properties (Mozyrska and Bartosiewicz, 2010; Mozyrska and Pawłuszewicz, 2010; Mozyrska et al., 2015; 2013b; Guermah et al., 2008b; Pawłuszewicz and Mozyrska, 2013; Balachandran and Kokila, 2012) and optimal control (Dzieliński, 2016; Bastos et al., 2011b; Bastos et al., 2011a; Trujillo and Ungureanu, 2018).

Various types of fractional difference operators have 
been introduced such as the Riemann-Liouville type difference operator, the Caputo type difference operator and the Grünwald-Letnikov type operator (Trujillo and Ungureanu, 2018). A more general $h$-difference operator has also been considered for these three types taking into account the presence of the sampling step $h$, which is useful in engineering applications (Mozyrska et al., 2015; 2013b; 2013a). In the present paper, the Grünwald-Letnikov type difference operator is considered.

Chaotic behavior of fractional-order systems has been extensively investigated in the literature. In the continuous-time domain, several fractional-order chaotic systems were introduced (see, e.g., the book by Petras (2011) and the references therein). Similarly, the study of the chaotic behavior fractional-order discrete-time systems was considered in the literature. Among the most important applications of fractional-order chaotic maps, one finds the fractional-order logistic map ( $\mathrm{Wu}$ and Baleanu, 2014b; Munkhammar, 2013) and the fractional-order Hénon map (Liu, 2014; Tarasov, 2010).

Due to their complex dynamics, chaotic systems experience widespread success in secure data communication (Feki et al., 2003; Luo and Wang, 2013; Pareek et al., 2006). In cryptosystems, the transmitter (the drive or master system) and the receiver (the response or slave system) are built as chaotic systems. The information to be secured is masked in the chaotic dynamics at the emitter level and then sent to the receiver through the public channel. At the receiver level, the secret information is decrypted. The use of fractional-order chaotic systems in secure data communication devices is very advantageous. Indeed, compared with integer-order chaotic dynamics, fractional-order chaotic dynamics are more complex because fractional-order derivatives have a complex geometric interpretation due to their non-local character and long memory (Podlubny, 2003). This complexity is an advantage in the design of secure communication schemes since it is difficult for an intruder to extract secret information hidden in fractional-order chaotic dynamics. In addition, the security key space is enlarged by fractional-order parameters, which therefore yields a high level of security.

From this perspective, the synchronization of fractional-order chaotic systems is a central problem. Inspired by the work of Pecora and Carrol (1990) and synchronization methods developed in the case of classical integer-order systems, synchronization of fractional-order chaotic systems in the continuous-time domain has received a lot of attention. Various methods such as the projective method, adaptive control, the impulsive method, active control and sliding mode techniques are proposed (Peng et al., 2014; Shao et al., 2016; Xi et al., 2014; Agrawal et al., 2012; Khanzadeh and Pourgholi, 2016).

On the other hand, the synchronization of fractional-order discrete-time systems is a very recent research topic despite its natural application in secure digitalized data communication such as image encryption (Zhen et al., 2012; Wu et al., 2016). Some works were devoted to this area (e.g., Wu and Baleanu, 2014a; Liao et al., 2013; Wua et al., 2016). As pointed out by Nijmeijer and Mareels (1997), synchronization may be viewed as an observer design problem. Moreover, in the context of secure communication, the reconstruction of the encrypted message is achieved within an unknown input observer (Sharma et al., 2016). The encrypted message is considered an unknown input. The objective of the unknown input observer is to estimate both the state and the unknown input.

In this paper, we investigative the synchronization of fractional-order discrete-time chaotic systems with the presence of unknown inputs. It is well known that observability is a fundamental property for estimating internal states on the basis of available input/output data (Nijmeijer, 1982; Hanba, 1982; Albertini and D'Alessandro, 2002). Also, the observability matching condition is another property when the system is subject to unknown inputs (Barbot et al., 2002). The problem of synchronization with recovery of the input can be seen as a left invertibility problem (Djemai et al., 2009). The observability of fractional-order discrete-time nonlinear systems described with the Grünwald-Letnikov type difference operator was studied by Mozyrska and Bartosiewicz (2010), who stated a rank condition. Similarly, the first aim of the present paper is to derive some new theoretical results on the observability and the observability matching condition of non-linear fractional-order discrete-time systems described by the Grünwald-Letnikov type difference operator. The concepts of output-memory indistinguishability and output-memory observability are introduced. Furthermore, rank criteria for output-memory observability and an output-memory observability matching condition are proposed.

In most works, synchronization is achieved asymptotically. It has been demonstrated in the case of non-linear integer-order discrete-time systems that synchronization can be immediate and accurate thanks to the use of an exact state reconstructor or a dead beat observer (Belmouhoub et al., 2003; Sira-Ramirez and Rouchon, 2001; Sira-Ramirez et al., 2002). In addition, the immediate recovery of encrypted information is a real challenge. The second contribution of this work is designing an exact state reconstructor for non-linear discrete-time fractional-order systems. The true state is recovered from a finite ring of delayed inputs and delayed outputs. The problem of the exact reconstruction of an unknown input is also considered. Exploiting 
these results, we propose a new chaotic encryption process to secure digital data communication based on fractional-order discrete-time chaotic systems. In the proposed secure data communication scheme, a fractional-order version of the generalized Hénon map (Richter, 2002) is used.

The outline of the paper is as follows. Section 2 is devoted to some definitions and preliminary results on fractional-order discrete-time system described by the Grünwald-Letnikov type operator. In Section 3, the concepts of output-memory indistinguishability and output-memory observability are introduced. Rank criteria for output-memory observability and the output-memory observability matching condition are established. The proposed exact delayed state reconstructor of a fractional order is developed in Section 4. With this tool, in Section 5, a secure data communication scheme based on the fractional-order generalized Hénon map is studied. Numerical simulations demonstrating the efficiency of the proposed approach are reported in Section 6. In Section 7, some conclusions and future perspectives end the paper.

\section{Preliminaries}

Let $h \in \mathbb{R}_{+}$be the sampling step and $a \in \mathbb{R}$ a fixed real number. Define the time scale as $(h \mathbb{N})_{a}=\{a, a+h, a+$ $2 h, \ldots, a+s h, \ldots\}$ with $s \in \mathbb{N}$, and denote by $\mathcal{F}$ the set of real-valued functions. Many fractional-order $h$-difference operator types have been introduced in the literature, namely, the fractional Riemann-Liouville type difference operator, the Caputo type difference operator and the Grünwald-Letnikov type difference operator (Atici and Eloe, 2007; 2009; Abdeljawad and Baleanu, 2009; Holm, 2011; Ortigueira, 2000; Chen et al., 2011; Mozyrska and Pawłuszewicz, 2011; 2012). In this paper, the Grünwald-Letnikov type- $h$-difference operator, defined below, is used.

Definition 1. Let $t \in(h \mathbb{N})_{a}$ and $\alpha \in \mathbb{R}_{+}$be a given arbitrary positive real number. The Grünwald-Letnikov type h-difference operator on a function $\nu(t) \in \mathcal{F}$ : $(h \mathbb{N})_{a} \rightarrow \mathbb{R}$ is defined as

$$
{ }_{a} \Delta_{h}^{\alpha} \nu(t)=\sum_{k=0}^{(t-a) / h} c_{k} \nu(t-k h),
$$

where $a$ denotes the initial time and

$$
c_{k}=\frac{1}{h^{\alpha}}(-1)^{k}\left(\begin{array}{l}
\alpha \\
k
\end{array}\right)
$$

with

$$
\left(\begin{array}{l}
\alpha \\
k
\end{array}\right)= \begin{cases}+1 & \text { for } k=0 \\
\frac{\alpha(\alpha-1)(\alpha-2) \ldots(\alpha-k+1)}{k !} & \text { for } k \geq 1\end{cases}
$$

Remark 1. The Grünwald-Letnikov $h$-difference operator on a given function $\nu(t)$ for $t \in \mathbb{R}$ is derived from the definition of the Grünwald-Letnikov fractional-order derivative

$$
\begin{aligned}
& G_{L} D_{t}^{\alpha} \nu(t) \\
& \quad=\lim _{h \rightarrow 0} \frac{1}{(h)^{\alpha}} \sum_{k=0}^{[(t-a) / h]}(-1)^{k}\left(\begin{array}{l}
\alpha \\
k
\end{array}\right) \nu(t-k h),
\end{aligned}
$$

where $[(t-a) / h]$ represents the integer part of $(t-a) / h$.

With no loss of generality, in what follows, the sampling time $h$ is taken as $h=1$ and the initial time as $a=0$. Indeed, in the context of designing a secure data communication scheme, the chaotic system at the emitter level is naturally discrete and is not derived from a sampling process. In this context, the step $h$ is therefore purely contrived. What is important is that the system acting as the emitter exhibits chaotic behavior.

In the works of Bastos et al. (2011a) as well as Ferreira and Torres (2011), the fractional difference operator is defined over the time scale $h \mathbb{N}$. This time scale is particularly advantageous in numerical solution of the optimal control problem for fractional-order continuous-time systems, because if $h$ tends to zero, the previous fractional continuous-time results are recovered (Bastos et al., 2011b; Ferreira and Torres, 2011). In addition, as pointed out by Ferreira and Torres (2011), the fractional-order difference operator with a given step $h$ different from 1 can be transformed to an equivalent operator with $h=1$. For simplicity, we use $\Delta^{\alpha}$ instead of ${ }_{0} \Delta_{1}^{\alpha}, \mathbb{N}$ instead of $(1 \mathbb{N})_{0}$ and $t \in \mathbb{N}$ as an integer number. If $h=1$, then $\left|c_{k}\right| \leq \alpha^{k} / k$ !, and thus the sequence $\left(c_{k}\right)$ is absolutely summable. Provided that the function $\nu(t)$ is uniformly bounded, the series (1) converges. It follows that $\Delta^{\alpha}$ is well defined.

Hereafter, the one unit delay operator $\delta$ is used in the sense that $\delta \nu(t)=\nu(t-1)$. Its inverse is denoted by $\delta^{-1}$ so that $\delta^{-1} \nu(t)=\nu(t+1)$. For any number $s \in \mathbb{N}$, $\delta^{s}$ and $\delta^{-s}$ stand for $\delta^{s} \nu(t)=\nu(t-s)$ and $\delta^{-s} \nu(t)=$ $\nu(t+s)$, respectively. The identity operator is denoted by $\delta^{0}=I_{d}$, i.e., $\delta^{0} \nu(t)=\nu(t)$. The delayed collection $\{\nu(t-1), \nu(t-2), \ldots, \nu(t-s)\}$ is represented by

$$
\underline{\delta}^{s} \nu(t)=\left\{\delta \nu(t), \delta^{2} \nu(t), \ldots, \delta^{s} \nu(t)\right\},
$$

and the forward shifts $\{\nu(t), \nu(t+1), \ldots, \nu(t+s)\}$ by

$$
\underline{\delta}^{-s} \nu(t)=\left\{\nu(t), \delta^{-1} \nu(t), \ldots, \delta^{-s} \nu(t)\right\} .
$$

Definition 2. Let $x(t) \in \mathcal{X} \in \mathbb{R}^{n}$ be an $n$-dimensional real-valued vector, where $\mathcal{X}$ denotes a differentiable manifold of dimension $n$. Denote by $x_{i}$, the $i$-th component of $x, i=1,2, \ldots, n$, say $x(t)=$ $\left[\begin{array}{llll}x_{1}(t) & x_{2}(t) & \ldots x_{n}(t)\end{array}\right]^{T}$, where $x_{i}(t) \in \mathcal{F}: \mathbb{N} \rightarrow$ $\mathbb{R}, i=1,2, \ldots, n$, are $n$ real-valued functions. Let 
$\alpha_{i} \in \mathbb{R}_{+}, i=1,2, \ldots, n$ be given real positive numbers. The Grünwald-Letnikov type difference operator on the $n$-dimensional vector $x(t) \in \mathcal{X}$ is defined as

$$
\begin{aligned}
& \Delta^{[\alpha]} x(t) \\
& \quad=\left[\begin{array}{lllll}
\Delta^{\alpha_{1}} x_{1}(t) & \Delta^{\alpha_{2}} x_{2}(t) & \ldots & \Delta^{\alpha_{n}} x_{n}(t)
\end{array}\right]^{T} .
\end{aligned}
$$

Similarly, the delay operator on the $n$-dimensional vector $x(t) \in \mathcal{X}$ is defined as

$$
\delta^{s} x(t)=\left[\begin{array}{llll}
\delta^{s} x_{1}(t) & \delta^{s} x_{2}(t) & \ldots & \delta^{s} x_{n}(t)
\end{array}\right]^{T} .
$$

Remark 2. In (2), if there exist a real number $\alpha>0$ and integers $k_{i}, i=1,2, \ldots, n$, such that all orders $\alpha_{i}$, $i=1,2, \ldots, n$, satisfy the relation

$$
\alpha_{i}=k_{i} \alpha, \quad i=1,2, \ldots, n,
$$

then the fractional-order difference operator $\Delta^{[\alpha]}$ is said to be a commensurate fractional-order difference operator of order $\alpha$ and is denoted simply by $\Delta^{\alpha}$ (Buslowicz, 2008). A particular case of an commensurate fractional-order difference operator is obtained when all orders $\alpha_{i}, i=$ $1,2, \ldots, n$, are equal, i.e., $k_{i}=1, i=1,2, \ldots, n$.

Definition 3. Denote by $\left(\Delta^{\alpha}\right)^{0}$, the identity operator, i.e., $\left(\Delta^{\alpha}\right)^{0} x(t)=x(t)$. The $\ell$ times iterated commensurate fractional-order difference operator of order $\alpha$ on a given $n$-dimensional real valued vector $x(t)$ for any integer number $\ell \in \mathbb{N}$ is defined as

$$
\left(\Delta^{\alpha}\right)^{\ell} x(t)=\Delta^{\alpha}\left(\Delta^{\alpha}\right)^{\ell-1} x(t), \quad \ell=1,2, \ldots
$$

or, equivalently,

$$
\left(\Delta^{\alpha}\right)^{\ell} x(t)=\sum_{k=0}^{t} c_{k}\left(\Delta^{\alpha}\right)^{\ell-1} x(t-k), \quad \ell=1,2, \ldots
$$

The next lemma provides a useful identity between the Grünwald-Letnikov type difference operator and the delay operator.

Lemma 1. Let $x(t)$ be an n-dimensional vector. For any given $\alpha \in \mathbb{R}_{+}$and any given integer $s \in \mathbb{N}$, we have the following commutative properties:

$$
\Delta^{\alpha}\left(\delta^{-s} x(t)\right)=\delta^{-s}\left(\Delta^{\alpha} x(t)\right)
$$

and, more generally,

$$
\left(\Delta^{\alpha}\right)^{\ell}\left(\delta^{-s} x(t)\right)=\delta^{-s}\left(\left(\Delta^{\alpha}\right)^{\ell} x(t)\right),
$$

$\ell=1,2, \ldots$

Proof. From (1), we have

$$
\Delta^{\alpha} x(t)=c_{0} x(t)+c_{1} x(t-1)+\cdots+c_{t} x(0) .
$$

It follows that

$$
\begin{aligned}
\delta^{-s} \Delta^{\alpha} x(t)= & c_{0} x(t+s)+c_{1} x(t+s-1) \\
& +\cdots+c_{t} x(s) .
\end{aligned}
$$

On the other hand, we can write

$$
\begin{aligned}
& \Delta^{\alpha} \delta^{-s} x(t) \\
& =\Delta^{\alpha} x(t+s) \\
& =c_{0} x(t+s)+c_{1} x(t+s-1)+\cdots+c_{t} x(s) .
\end{aligned}
$$

Clearly, (8) and (9) imply

$$
\Delta^{\alpha}\left(\delta^{-s} x(t)\right)=\delta^{-s}\left(\Delta^{\alpha} x(t)\right) .
$$

The relation (7) is obtained by induction. This completes the proof.

\section{Observability of nonlinear discrete-time fractional-order systems}

Observability is a crucial property in systems theory. It informs us about the possibility of constructing a system capable of estimating internal state variables from input-output data. The unavailability of state variables requires the use of reconstruction methods of state variables such as state observers. Observability and observer design of fractional-order systems were investigated by several authors (Kaczorek, 2016; N'Doye et al., 2016). For linear discrete-time systems, the concept of observability was introduced and some observability criteria were proposed (Mozyrska and Bartosiewicz, 2010; Moryrska and Pawłuszkiewicz 2010; Mozyrska et al., 2015; 2013b; Guermah et al., 2008b; Pawłuszewicz and Mozyrska, 2013) and observability with finite initial memory was introduced by Mozyrska and Pawłuszewicz (2010) as well as Mozyrska et al. (2015). However, for fractional-order non-linear discrete-time systems, to the best of our knowledge, only Mozyrska and Bartosiewicz (2010) formally defined the concept of observability.

In the following, this idea is extended, a new concept of output-memory observability is introduced, and a new observability criterion for non-linear discrete-time fractional-order systems is proposed.

Consider the following fractional-order nonlinear discrete-time system:

$$
\begin{aligned}
\Delta^{\alpha} x(t+1) & =f(x(t), u(t)), \quad x(0)=x_{0}, \\
y(t) & =h(x(t)),
\end{aligned}
$$

where $x(t) \in \mathcal{X} \subset \mathbb{R}^{n}$ is the state vector, $x_{0}$ is the initial condition, $u(t) \in \mathcal{U} \subset \mathbb{R}$ stands for the control input and $y(t) \in \mathcal{Y} \subset \mathbb{R}$ signifies the measured output, where $\mathcal{X}$ and $\mathcal{Y}$ are differentiable manifolds of dimension $n$ and 1 , respectively, and $\mathcal{U}$ denotes an open control interval in $\mathbb{R}$. The system 110 is assumed to be of class $\mathcal{C}^{\infty}$, 
i.e., the functions $f: \mathcal{X} \times \mathcal{U} \rightarrow \mathcal{X}$ are of class $\mathcal{C}^{\infty}$. We assume that the system evolves around an equilibrium point $\left(x_{e}, u_{e}, y_{e}\right), x_{e} \in \mathcal{X}$ defined as follows.

Definition 4. We say that $\left(x_{e}, u_{e}, y_{e}\right)$ is an equilibrium point of 10] if

$$
\begin{aligned}
\Delta^{\alpha} x_{e} & =f\left(x_{e}, u_{e}\right)=x_{e}, \\
y_{e} & =h\left(x_{e}\right) .
\end{aligned}
$$

For convenience, we denote by $f_{u}: \mathcal{X} \rightarrow \mathcal{X}$ the map $f_{u}(x(t))=f(x(t), u(t))$ and by $f_{\left(\Delta^{\alpha}\right)^{\ell} u}: \mathcal{X} \rightarrow \mathcal{X}$ the $\operatorname{map} f_{\left(\Delta^{\alpha}\right)^{\ell} u}(x(t))=f\left(x(t),\left(\Delta^{\alpha}\right)^{\ell} u(t)\right), \ell=1,2, \ldots$ Moreover, we write $f_{u_{j}}$ and $f_{\left(\Delta^{\alpha}\right)^{\ell} u_{j}}$ for $f(x(t), u(j))$ and $f\left(x(t),\left(\Delta^{\alpha}\right)^{\ell} u(j)\right), j=0,1,2, \ldots$, respectively. Below, the classical definitions of indistinguishability and observability are revisited in the context of fractional-order non-linear discrete-time systems. More precisely, we introduce the concept of the output-memory observability of a state $x^{0}$ as the possibility to distinguish the state $x^{0}$ from any other state $x^{j}, j \neq 0$, by applying a finite control sequence $U_{k}=\{u(0), u(1), \ldots, u(k)\}$ and scanning the corresponding memory of the output $Y_{m k}=\left\{y(0), \Delta^{\alpha} y(1), \ldots,\left(\Delta^{\alpha}\right)^{k} y(k)\right\}$.

Definition 5. Two states $x^{1}, x^{2} \in \mathcal{X}$ are said to be outputmemory indistinguishable if, for each control sequence $U_{k}=\{u(0), u(1), \ldots, u(k)\}$ and for each $k \geq 0$, the produced output memories are identical, i.e.,

$$
\begin{aligned}
& h \circ f_{\left(\Delta^{\alpha}\right)^{k} u_{k}} \circ f_{\left(\Delta^{\alpha}\right)^{k-1} u_{k-1}} \circ \ldots f_{\Delta^{\alpha} u_{1}} \circ f_{u_{0}}\left(x^{1}\right) \\
= & h \circ f_{\left(\Delta^{\alpha}\right)^{k} u_{k}} \circ f_{\left(\Delta^{\alpha}\right)^{k-1} u_{k-1}} \circ \ldots f_{\Delta^{\alpha} u_{1}} \circ f_{u_{0}}\left(x^{2}\right),
\end{aligned}
$$

where "o" is the composition operator.

Denote by $x I x^{0}$ the set of all states $x$ indistinguishable from $x^{0}$. The concept of outputmemory observability is defined below.

Definition 6. A state $x^{0}$ is said to be output-memory observable if $x I x^{0}=\left\{x^{0}\right\}, \forall x \in \mathcal{X}$.

Remark 3. As for integer-order systems, local weak and strong observability properties can be defined. We say that a state $x^{0}$ is locally weakly output-memory $o b$ servable if there exists a neighbourhood $\mathcal{W}_{x_{0}} \subset \mathcal{X}$ of $x^{0}$ such that, for any state $x \in \mathcal{W}_{x_{0}}$, we have $x I x^{0}=\left\{x^{0}\right\}$, $\forall x \in \mathcal{W}_{x_{0}}$. Similarly, a state $x^{0}$ is locally strongly output-memory observable if there exists a neighbourhood $\mathcal{W}_{x_{0}} \subset \mathcal{X}$ of $x^{0}$ such that, for each $x^{1}, x^{2} \in \mathcal{W}_{x_{0}}, x^{1} I x^{2}$ implies that $x^{1}=x^{2}$.

Definition 7. The fractional-order non-linear discrete-time system (10) is output-memory observable if each state $x \in \mathcal{X}$ is output-memory observable.
For integer-order nonlinear discrete-time systems, the notion of observability is often related to the injectivity of an observation map and the full rank of its Jacobian (Nijmeijer, 1982; Hanba, 1982; Albertini and D'Alessandro, 2002). Mimicking these results, the output-memory observability criterion of the fractional-order non-linear discrete-time system 10 is proposed below. Define the observability matrix as follows:

$$
\mathcal{O}_{n-1}(x)=\left(\begin{array}{c}
\mathrm{d} h(x(t)) \\
\mathrm{d} h \circ f_{u}(x(t)) \\
\mathrm{d} h \circ f_{\Delta^{\alpha} u} \circ f_{u}(x(t)) \\
\vdots \\
\mathrm{d} h \circ f_{\left(\Delta^{\alpha}\right)^{(n-2)} u} \circ \ldots \circ f_{u}(x(t))
\end{array}\right),
$$

where $d(\ldots)$ stands for the standard differential operator with respect to $x$, i.e., $\mathrm{d} \mu(x)=\left[\frac{\partial \mu(x)}{\partial x_{1}} \ldots \frac{\partial \mu(x)}{\partial x_{n}}\right]$ for any real-valued function $\mu(x)$. As in the integer-order case, the dimension $\mathcal{O}_{n-1}(x)$ may be non-constant in the domain considered and may decrease (Albertini and D’Alessandro, 1996; Jakubczyk and Sontag, 1990). A strong result of output-memory observability can be derived under the following assumption.

Assumption 1. The observability matrix $\mathcal{O}_{n-1}(x)$ is a constant dimension in a neighbourhood of $\mathcal{W}_{x_{e}} \in \mathcal{X}$ of an equilibrium point $x_{e}$.

Theorem 1. Let Assumption 1 be satisfied. The fractional-order non-linear discrete-time system (10) is locally output-memory observable in a neighbourhood $\mathcal{W}_{x_{e}} \in \mathcal{X}$ of an equilibrium point $x_{e}$ if and only if the following rank condition holds:

$$
\operatorname{rank}\left(\mathcal{O}_{n-1}(x)\right)=n
$$

for all $x \in \mathcal{W}_{x_{e}}$

Proof. The proof consists in constructing an appropriate observability map $\Theta_{n-1}(x)$ from the input and output data. From system equations and Lemma 1, the following relation is obtained:

$$
\left(\Delta^{\alpha}\right)^{\ell} \delta^{-\ell} y(t)=h \circ f_{\left(\Delta^{\alpha}\right)^{(\ell-1)} u} \circ \ldots \circ f_{u}(x(t)),
$$

$\ell=1,2, \ldots$ Then the observability map is constructed as

$$
\Theta_{n-1}(x)=\left(\begin{array}{c}
y(t) \\
\Delta^{\alpha} \delta^{-1} y(t) \\
\left(\Delta^{\alpha}\right)^{2} \delta^{-2} y(t) \\
\vdots \\
\left(\Delta^{\alpha}\right)^{n-1} \delta^{-(n-1)} y(t)
\end{array}\right)
$$




$$
=\left(\begin{array}{c}
h(x(t)) \\
h \circ f_{u}(x(t)) \\
h \circ f_{\Delta^{\alpha} u} \circ f_{u}(x(t)) \\
\vdots \\
h \circ f_{\left(\Delta^{\alpha}\right)^{(n-2)} u} \circ \ldots \circ f_{u}(x(t))
\end{array}\right) .
$$

The observability matrix is given by the co-distribution of the observability map, i.e., $\mathcal{O}_{n-1}=\mathrm{d} \Theta_{n-1}$. By Assumption 1, the observability matrix $\mathcal{O}_{n-1}(x)$ is a constant dimension in a neighbourhood of $\mathcal{W}_{x_{e}} \in \mathcal{X}$, i.e., $\operatorname{dim} \mathcal{O}_{n-1}(x)=n$ for a all $x \in \mathcal{W}_{x_{e}}$. Then, with this assumption, if the observability matrix $\mathcal{O}_{n-1}(x)$ is of full rank, Eqn. (15) possesses a unique solution $x(t)$. This completes the proof.

Remark 4. Note that the observability map $\Theta_{n-1}(x)$ and the observability matrix $\mathcal{O}_{n-1}(x)$ depend on the input $u$. The fractional-order nonlinear discrete-time system (10) is uniformly output-memory observable in a neighbourhood $\mathcal{W}_{x_{e}} \in \mathcal{X}$ if the rank condition (14) is satisfied for all $u \in \mathcal{U} \subset \mathbb{R}$.

Another property of interest is the possibility of recovering not only the state but also the input assumed to be unknown from the observation of the output. This property is connected to the left-invertibility problem (Djemai et al., 2009). The left-invertibility property is guaranteed by the so-called observability matching condition (Barbot et al., 2002). For this problem, we consider the class of fractional-order discrete-time input affine non-linear system described by

$$
\begin{gathered}
\Delta^{\alpha} x(t+1)=f(x(t))+g(x(t)) u(t), \quad x(0)=x_{0}, \\
y(t)=h(x(t))
\end{gathered}
$$

We assume that the input $u(t) \in \mathbb{R}$ is unknown. Before addressing the observability matching condition, the relative degree of the fractional-order non-linear discrete-time system (16) should be defined.

Definition 8. The relative degree of the fractional-order nonlinear discrete-time system (16) with respect to its input $u(t)$ is the smallest required number $r$ such that the output quantity $\left(\Delta^{\alpha}\right)^{r} \delta^{-r} y(t)$ depends explicitly on $u(t)$.

Definition 9. We say that the system 16ulfils the output-memory observability matching condition if $y(t), \Delta^{\alpha} \delta^{-1} y(t), \ldots,\left(\Delta^{\alpha}\right)^{(n-1)} \delta^{-(n-1)} y(t)$ does not depend on the unknown input while, in contrast, $\left(\Delta^{\alpha}\right)^{(n)} \delta^{-(n)} y(t)$ depends on the unknown input $u(t)$. Equivalently, the system (16) satisfies the output-memory observability matching condition if its relative degree is equal to $n$.

Accordingly, we have the following proposition.

Proposition 1. The system (16) satisfies the locally output-memory observability matching condition for $x \in$

$$
\mathcal{W}_{x_{e}} \subset \mathcal{X} \text { if }
$$

$$
\mathcal{O}_{n-1}(x) g(x)=\left(\begin{array}{c}
0 \\
0 \\
\vdots \\
0 \\
\pi(x)
\end{array}\right), \quad \begin{aligned}
& \\
& \forall x \in \mathcal{W}_{x_{e}} \subset \mathcal{X},
\end{aligned}
$$

where $\pi(x)$ is any non-vanishing function of $x \in \mathcal{W}_{x_{e}} \subset$ $\mathcal{X}$.

This output-memory observability matching condition allows recovering the unknown input $u(t)$ from a finite sequence of past values of the output $y(t)$.

\section{Exact state reconstructor}

In this section, we propose an exact delay reconstructor which guarantees an exact recovery of the true state provided that a finite string of past inputs applied and the produced outputs is available. In the first part of this section, we assume that the input is known and only the reconstruction of the state matters. The exact delayed reconstruction of the true state is announced in the following proposition.

Proposition 2. Assume that the system (10) is outputmemory observable in a domain $\mathcal{W}_{x_{e}} \in \mathcal{X}$ around an equilibrium point $\left(x_{e}, u_{e}, y_{e}\right)$. Then the fractional-order non-linear discrete-time system (10) is reconstructible in $\mathcal{W}$, i.e., the state $x(t)$ at time $t$ is exactly expressed in terms of the input and output data collections $\underline{\delta}^{n-1} u(t)$ and $\underline{\delta}^{n-1} y(t)$, respectively, provided that these collections are completely known.

Proof. From Theorem 1, there exists a mapping $\Psi$ such that the state $x(t)$ at each instant $t$ can be expressed by the increments in the outputs and the inputs as

$$
x(t)=\Psi\left(\underline{\delta}^{-(n-1)} y(t), \underline{\delta}^{-(n-2)} u(t)\right) .
$$

It follows that

$$
\delta^{n-1} x(t)=\Psi\left(y(t), \underline{\delta}^{(n-1)} y(t), \underline{\delta}^{(n-1)} u(t)\right),
$$

which means that $\delta^{n-1} x(t)$ can be expressed by the past output and input data. On the other hand, from the system equation (10), expanding $\Delta^{\alpha} x(t+1)$ yields

$$
\begin{aligned}
x(t+1) & =f(x(t), u(t))-m_{x}(t) \\
& =F(x(t), u(t)),
\end{aligned}
$$

where $m_{x}(t)$ is the accumulated memory of the state given by

$$
m_{x}(t)=\sum_{j=0}^{t}(-1)^{j+1} c_{j+1} x(t-j) .
$$


Iterating (20), we obtain

$$
x(t)=F^{(n-1)}\left(\delta^{n-1} x(t), \underline{\delta}^{(n-1)} u(t)\right),
$$

where $F^{(n-1)}$ obeys the recursion

$$
\begin{gathered}
F^{(k)}(\cdot)=F\left(F^{(k-1))}(\cdot), \quad \forall k=2,3, \ldots,\right. \\
F^{(1)}(\cdot)=F(\cdot)
\end{gathered}
$$

Substituting (19) on the right-hand side of (22) leads to the following relation:

$$
x(t)=\phi\left(y(t), \underline{\delta}^{(n-1)} y(t), \underline{\delta}^{(n-1)} u(t)\right) .
$$

Clearly, the true state $x(t)$ can be exactly reconstructed from the delayed input and output data. This completes the proof.

In the second part of this section, we discuss the problem of reconstructing both the system state and input. For this problem, we consider the observable form of (16),

$$
\begin{aligned}
\Delta^{\alpha} x(t+1) & =A x(t)+\Gamma(x(t))+g(x) u(t), \\
y(t) & =C x(t) .
\end{aligned}
$$

The system matrices $(A, C)$ take the following Brunowski form:

$$
\begin{aligned}
A & =\left[\begin{array}{ccccc}
0 & 1 & 0 & \ldots & 0 \\
0 & 0 & 1 & 0 & \vdots \\
\vdots & \vdots & \vdots & \vdots & \vdots \\
0 & 0 & 0 & \ldots & 0
\end{array}\right], \\
C & =\left[\begin{array}{llll}
1 & 0 & \ldots & 0
\end{array}\right] .
\end{aligned}
$$

The non-linear vector function $\Gamma(x(t))$ possesses the following triangular structure:

$$
\Gamma(x)=\left(\begin{array}{c}
\Gamma_{1}\left(x_{1}\right) \\
\Gamma_{2}\left(x_{1}, x_{2}\right) \\
\Gamma_{3}\left(x_{1}, x_{2}, x_{3}\right) \\
\vdots \\
\Gamma_{n}\left(x_{1}, x_{2}, x_{3}, \ldots, x_{n}\right)
\end{array}\right),
$$

and the vector field $g(x)$ is given by

$$
g(x)=\left(\begin{array}{c}
0 \\
0 \\
0 \\
\vdots \\
b_{n}(x)
\end{array}\right)
$$

with $b_{n}(x) \neq 0 \forall x(t) \in \mathcal{W}_{x_{e}} \subset \mathcal{X}$. This means that the unknown input affects only the dynamics of the last variable $x_{n}(t)$.

In general, a given system is not in this particular form. However, if this system satisfies the output-memory observability condition, this form can be obtained after a suitable change of coordinates (Barbot et al., 2002; Djemai et al., 2009). With this form, the observability map $\Theta_{n-1}(x)$ does not depend on the unknown input $u$. This implies that the state $x(t)$ in (23) does not depend on the input, i.e.,

$$
x(t)=\phi\left(y(t), \underline{\delta}^{(n-1)} y(t)\right) .
$$

The state $x(t)$ is reconstructed uniquely from the output data. The relative degree is equal to $n$. This means that the input $u(t)$ appears explicitly in $\left(\Delta^{\alpha}\right)^{(n)} \delta^{-(n)} y(t)$, i.e.,

$$
\left(\Delta^{\alpha}\right)^{(n)} \delta^{-(n)} y(t)=\tilde{\Gamma}(x(t))+b_{n}(x(t)) u(t),
$$

where $\tilde{\Gamma}(x(t))$ is the compound function of $\Gamma_{i}(x(t))$, $i=1,2, \ldots, n$. The relation (28) means that the input depends on the advances of the output. It follows that, if $b(x) \neq 0$, for all $x(t) \in \mathcal{W}_{x_{e}} \subset \mathcal{X}$, the unknown input $u(t)$ can be exactly recovered after $n$ backward shifts from the reconstructed state $x(t)$ and the past output data.

\section{Exact synchronization of the fractional-order chaotic generalized Hénon map for secure communication}

The Hénon map (Hénon, 1976) has been widely studied to illustrate the chaotic behaviors of integer-order discrete-time non-linear systems. The map depends on parameters two $a$ and $b(a \neq 0, b \neq 0)$, and is described by the two following difference equations:

$$
\begin{aligned}
& x_{1}(t+1)=a-x_{1}^{2}(t)-b x_{2}(t), \\
& x_{2}(t+1)=x_{1}(t) .
\end{aligned}
$$

A generalized Hénon map was proposed by Richter (2002). It is described by the following difference equations:

$$
\begin{aligned}
& x_{1}(t+1)=a-x_{n-1}^{2}(t)-b x_{n}(t), \\
& x_{i}(t+1)=x_{i-1}(t), \quad i=2,3, \ldots, n .
\end{aligned}
$$

The equivalent commensurate fractional-order generalized Hénon map is written as

$$
\begin{aligned}
\Delta^{\alpha} x_{1}(t+1) & =a-x_{1}(t)-x_{n-1}^{2}(t)-b x_{n}(t), \\
\Delta^{\alpha} x_{i}(t+1) & =x_{i-1}(t)-x_{i}(t), \quad i=2,3, \ldots, n .
\end{aligned}
$$

In what follows, a three-dimensional fractional-order generalized Hénon map is considered to built a secure communication scheme. The transmitter is given by the driven (master) system

$$
\begin{aligned}
& \Delta^{\alpha} x_{1}(t+1)=a-x_{1}(t)-x_{2}^{2}(t)-b x_{3}(t), \\
& \Delta^{\alpha} x_{2}(t+1)=x_{1}(t)-x_{2}(t), \\
& \Delta^{\alpha} x_{3}(t+1)=x_{2}(t)-x_{3}(t)+w(t),
\end{aligned}
$$


where $w(t)$ represents a confidential message to be securely transmitted to the receiver. This message is modulated into chaotic system dynamics.

\subsection{Chaotic behaviour of the fractional-order} Hénon map. Chaotic behaviour of discrete-time fractional-order systems has been investigated recently by many authors. Generally, the main tools used to confirm the strange chaotic attractor are the phase portrait, the bifurcation diagram, and the computation of the Lyapunov exponents. It must be emphasized that there are major differences between integer-order chaotic maps and the corresponding fractionalized maps.

Despite the difficulties brought about by the complex aspect of fractional-order systems, these tools have been successfully exploited. For instance, the chaotic behaviour of the fractional-order Hénon map has been proven by using the bifurcation diagram and phase portraits (Liu, 2014; Tarasov, 2010). Similarly in the works of $\mathrm{Wu}$ and Baleanu (2014b) as well as Munkhammar (2013), the bifurcation diagram and the phase portrait of the fractional-order logistic map were used to detect the chaotic behaviour. Wu and Baleanu (2015) investigated the Lyapunov exponent analysis for the fractional-order logistic map and the fractional-order delayed logistic map.

As is mentioned by $\mathrm{Wu}$ and Baleanu (2015), the computation of the Lyapunov exponents for fractional-order maps is difficult because the Jacobian matrix cannot be directly obtained. Indeed, the current state of the fractional-order system depends on all past states as given by Eqn. (20). Because of this accumulated memory effect, the Jacobian matrix algorithm (Wolf et al., 1985; Eckmann and Ruelle, 1985) is no longer possible. A tangent map based on the Volterra solution was proposed by $\mathrm{Wu}$ and Baleanu (2015). Even in this representation, the memory, effect is still present.

In order to make the calculations more accessible, we reduce the length of memory which is called the short memory principle in the literature (Podlubny, 1998). It is obvious that the system with the reduced memory is a new one, whose behaviour is different from the original system. The short memory system without the message is given in the following explicit form:

$$
\begin{aligned}
x_{1}(t+1)= & a+(\alpha-1) x_{1}(t)-x_{2}^{2}(t)-b x_{3}(t) \\
& -\sum_{k=1}^{L} c_{k+1} x_{1}(t-k), \\
x_{2}(t+1)= & x_{1}(t)+(\alpha-1) x_{2}(t) \\
& -\sum_{k=1}^{L} c_{k+1} x_{2}(t-k),
\end{aligned}
$$

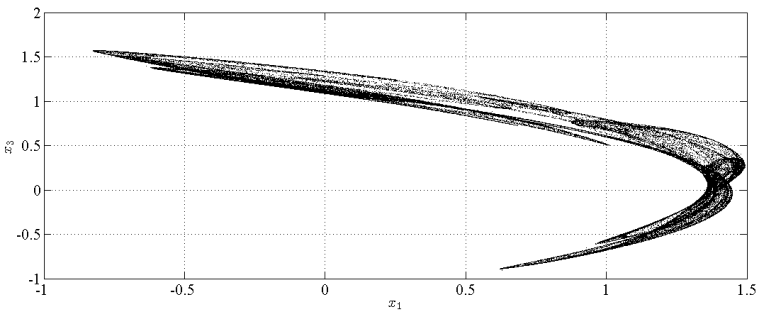

Fig. 1. Strange attractor for the fractional-order generalized discrete Hénon map in the $\left(x_{1}, x_{3}\right)$-plane.
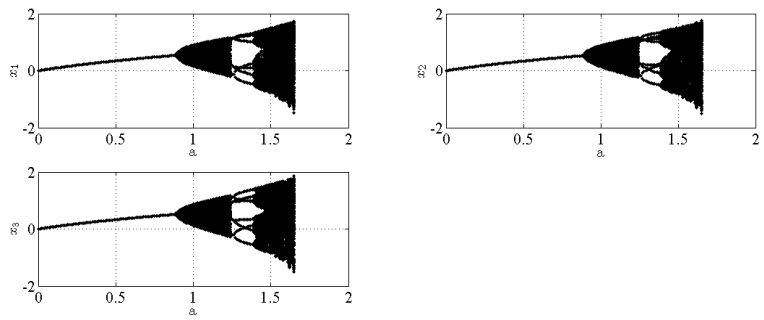

Fig. 2. Bifurcation diagrams versus the parameter $a$ of the fractional-order generalized discrete Hénon map for $\alpha=$ $0.9, b=0.1$ and $L=5$.

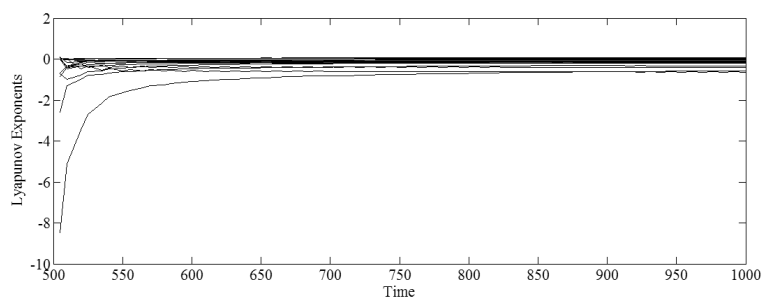

Fig. 3. Lyapunov exponents of the fractional-order generalized discrete Hénon map for $\alpha=0.9, a=1.5, b=0.1$ and $L=5$.

$$
\begin{aligned}
x_{3}(t+1)= & x_{2}(t)+(\alpha-1) x_{3}(t) \\
& -\sum_{k=1}^{L} c_{k+1} x_{3}(t-k),
\end{aligned}
$$

where $L$ denotes the memory length.

The use of this new system is highly interesting in the design of secure communication schemes. In fact, as we will see in the next section, the length $L$ of the memory is an additional parameter of the security key. The chaotic behaviour of the new system depends on the parameters but also the length of the memory. Note that the stability and trajectory boundedness depend on both $L$ and $\alpha$, as reported by Dzieliński and Sierociuk (2008) or Edelman (2018).

In what follows, the chaotic behaviour of the fractional-order map (34) is analyzed. The phase portrait, the bifurcation diagram and the Lyapunov exponents are successively used. The phase portrait is plotted in Fig. 1. The simulations performed for the values of the system parameters taken as $\alpha=0.9, a=1.5, b=0.1$ and 
$L=5$ indicate that the fractional-order system presents chaotic behaviour. Indeed, Fig. 1 plots the strange chaotic attractor of the three-dimensional fractional-order generalized Hénon map on the $\left(x_{1}, x_{3}\right)$ plane.

Bifurcation diagrams versus the parameter $a$ are plotted in Figs. 2(a)-(c) for $\alpha=0.9, b=0.1$ and $L=5$. From these figures, we deduce that the chaos phenomenon appears for $a=1.5$. Other simulations not reported here show that the chaotic behaviour still appears for higher values of $L$, while other parameters remain unchanged.

Finally, the Lyapunov exponents method is investigated. To apply the classical Jacobian matrix algorithm (Wu and Baleanu, 2015), the short-memory system (34) for $L=5$ is rewritten in augmented form as follows. Define new state variables $z_{i}^{j}(t)=x_{i}(t-j)$, $i=1,2,3$ and $j=1,2,3,4,5$. Then the system (34) becomes

$$
\begin{aligned}
z_{1}^{1}(t+1)= & a+(\alpha-1) z_{1}^{1}(t)-\left(z_{2}^{1}\right)^{2}(t)-b z_{3}^{1}(t) \\
& -\sum_{j=1}^{5} c_{j+1} z_{1}^{j}(t), \\
z_{2}^{1}(t+1)= & z_{1}^{1}(t)+(\alpha-1) z_{2}^{1}(t) \\
& -\sum_{j=1}^{5} c_{j+1} z_{2}^{j}(t), \\
z_{3}^{1}(t+1)= & z_{2}^{1}(t)+(\alpha-1) z_{3}^{1}(t) \\
& -\sum_{j=1}^{5} c_{j+1} z_{3}^{j}(t), \\
z_{i}^{j}(t+1)= & z_{i}^{j-1}(t), \quad i=1,2,3, \quad j=2,3,4,5 .
\end{aligned}
$$

Figure 3 plots the Lyapunov exponents for $\alpha=0.9$, $a=1.5, b=0.1$ and $L=5$. The eighteen Lyapunov exponents are 0.079277, 0.035635, -0.0045199, $-0.045184, \quad-0.062916, \quad-0.089242, \quad-0.093286$, $-0.10464,-0.13501,-0.16417,-0.17261,-0.1804$, $-0.18501,-0.19651,-0.32537,-0.39704,-0.572424$, -0.6341 . The system possesses two positive Lyapunov exponents. Then, it is hyperchaotic. This confirms the chaotic behaviour.

5.2. Observability and observer design. We use single channel communication. The only information to be transmitted to the receiver is taken as $y(t)=x_{2}(t)$. In order to develop the observer, the output-memory $o b$ servability property and the output-memory observability matching condition should be satisfied. The system (33) with the output $y(t)=x_{2}(t)$ is written as

$$
\begin{aligned}
\Delta^{\alpha} x(t+1) & =f(x(t))+g(x(t)) w(t), \\
y(t) & =h(x),
\end{aligned}
$$

where $x(t)=\left[\begin{array}{lll}x_{1}(t) & x_{2}(t) & x_{3}(t)\end{array}\right]^{T}, h(x)=x_{2}(t)$ and

$$
\begin{aligned}
& f(x)=\left(\begin{array}{c}
a-x_{1}(t)-x_{2}^{2}(t)-b x_{3}(t) \\
x_{1}(t)-x_{2}(t) \\
x_{2}(t)-x_{3}(t)
\end{array}\right), \\
& g(x)=\left(\begin{array}{l}
0 \\
0 \\
1
\end{array}\right) .
\end{aligned}
$$

From the relations

$$
\begin{aligned}
y(t) & =h(x(t))=x_{2}(t), \\
\Delta^{\alpha} \delta^{-1} y(t) & =h \circ f(x(t))=\Delta^{\alpha} x_{2}(t+1) \\
& =-x_{2}(t)+x_{1}(t), \\
\Delta^{\alpha(2)} \delta^{-2} y(t) & =h \circ f^{(2)}(x(t)) \\
& =-\Delta^{\alpha} x_{2}(t+1)+\Delta^{\alpha} x_{1}(t+1) \\
& =-2 x_{1}(t)+a+x_{2}(t)-x_{2}^{2}(t)-b x_{3}(t),
\end{aligned}
$$

the observability matrix of (33) is deduced as

$$
\begin{aligned}
\mathcal{O}_{2}(x) & =\left(\begin{array}{c}
\mathrm{d} h(x(t)) \\
\mathrm{d} h \circ f(x(t)) \\
\mathrm{d} h \circ f^{(2)}(x(t))
\end{array}\right) \\
& =\left[\begin{array}{ccc}
0 & 1 & 0 \\
1 & -1 & 0 \\
-2 & 1-2 x_{2} & -b
\end{array}\right] .
\end{aligned}
$$

Since $\operatorname{det}\left(\mathcal{O}_{2}(x)\right)=b \neq 0$, the system (33) is output-memory observable for all $x(t) \in \mathbb{R}^{3}$. The outputmemory observability matching condition can also be checked. Indeed, we have

$$
\mathcal{O}_{2}(x) g(x)=\left[\begin{array}{c}
0 \\
0 \\
-b
\end{array}\right] .
$$

Since $b \neq 0$, the output-memory observability matching condition is satisfied for all $x(t) \in \mathbb{R}^{3}$. As mentioned by Nijmeijer and Mareels (1997), synchronization of a chaotic system can be formulated as an observer design problem. The receiver is described by the following response (slave) system:

$$
\begin{aligned}
\Delta^{\alpha} \hat{x}_{1}(t+1) & =a-\hat{x}_{1}(t)-y^{2}(t)-b \hat{x}_{3}(t), \\
\Delta^{\alpha} \hat{x}_{2}(t+1) & =\hat{x}_{1}(t)-y(t), \\
\Delta^{\alpha} \hat{x}_{3}(t+1) & =y-\hat{x}_{3}(t),
\end{aligned}
$$

where $\hat{x}_{1}, \hat{x}_{2}$ and $\hat{x}_{3}$ are the estimates of the driven state variables $x_{1}, x_{2}$ and $x_{3}$, respectively.

Without any additional control signal injection, except the transmitted signal $y(t)$, we show that this observer permits us to exactly reconstruct the true variables $x_{i}(t), i=1 ; 2,3$, and to find the original 
message $w(t)$. Subtracting (36) from (33) yields the dynamic error given by

$$
\begin{aligned}
& \Delta^{\alpha} e_{1}(t+1)=-e_{1}(t)-b e_{3}(t), \\
& \Delta^{\alpha} e_{2}(t+1)=e_{1}(t), \\
& \Delta^{\alpha} e_{3}(t+1)=-e_{3}(t)+w(t),
\end{aligned}
$$

where $e_{i}(t)=x_{i}(t)-\hat{x}_{i}(t), i=1,2,3$, are the estimation errors. Since $y(t)=x_{2}(t)$ is known from the receiver, $e_{2}(t)$ is known at each instant $t$. We can then consider $e_{2}(t)$ to be an output of the estimation errors system (37), i.e.,

$$
e_{y}(t)=e_{2}(t)=x_{2}(t)-\hat{x}_{2}(t)=y(t)-\hat{y}(t) .
$$

The objective is to find the secret message $w(t)$. By applying the exact delayed recontructor developed in the previous section for (37), the errors $e_{1}(t)$ and $e_{3}(t)$ are determined from $e_{3}(t)$, and the message $w(t)$ is also found. The error system (37) is written as

$$
\begin{aligned}
e_{1}(t+1)= & (\alpha-1) e_{1}(t)-b e_{3}(t)-m_{e 1}(t-1), \\
& e_{y}(t+1)=e_{1}(t)-m_{e y}(t), \\
e_{3}(t+1)= & (\alpha-1) e_{3}(t)-m_{e 3}(t-1)+w(t),
\end{aligned}
$$

with

$$
\begin{gathered}
m_{e i}(t-1)=\sum_{k=1}^{t} c_{k+1} e_{i}(t-k), \quad i=1,3, \\
m_{e y}(t)=\sum_{k=0}^{t} c_{k+1} e_{y}(t-k) .
\end{gathered}
$$

From 40), we have

$$
e_{1}(t)=e_{y}(t+1)+m_{e y}(t) .
$$

Since $e_{y}(t+1)$ is not available, we must delay (42) by one time step; then

$$
e_{1}(t-1)=e_{y}(t)+m_{e y}(t-1) .
$$

This means that $e_{1}(t)$ can be known after one backward shift. This allows us to reconstruct the state variable $x_{1}(t)$ after one backward shift. Applying two backward shifts for (39), we get

$$
\begin{aligned}
e_{1}(t-1)= & (\alpha-1) e_{1}(t-2)-b e_{3}(t-2) \\
& -m_{e 1}(t-3) .
\end{aligned}
$$

Since $e_{1}(t-1), e_{1}(t-2)$ and, obviously, $m_{e 1}(t-$ $3)$ are known, $e_{3}(t-2)$ can be obtained. This allows us to reconstruct $x_{3}(t)$ after two backward shifts. Now, applying three backward shifts to the last equation (41), we get

$$
e_{3}(t-2)=(\alpha-1) e_{3}(t-3)-m_{e 3}(t-4)+w(t-3) .
$$

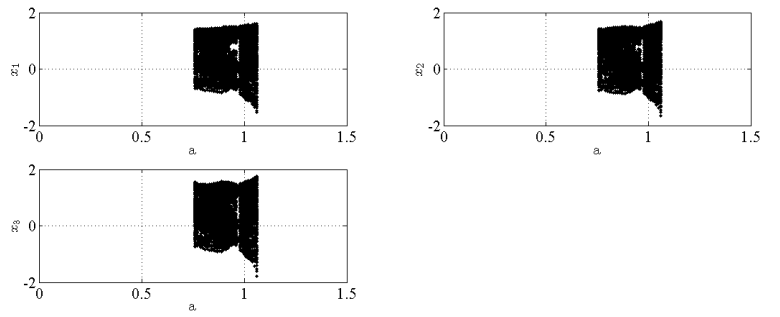

Fig. 4. Bifurcation diagrams versus the order $\alpha$ of the fractional-order generalized discrete Hénon map for $a=$ $1.5, b=0.1$ and $L=5$.

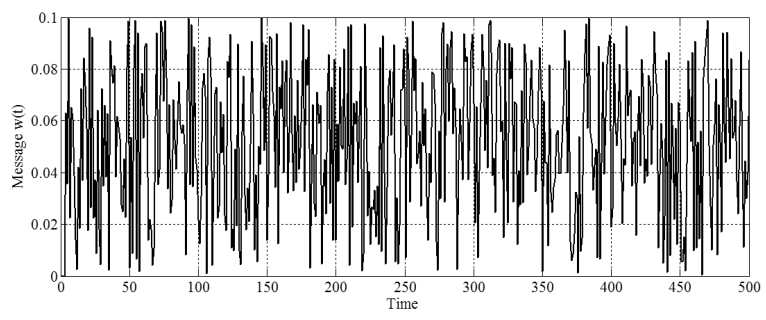

Fig. 5. Message $w(t)=0.1 \operatorname{rand}(500,1)$.

Again here, since $e_{3}(t-2), e_{3}(t-3)$ and $m_{e 1}(t-4)$ are known, it follows that $w(t-3)$ can be recovered. Accordingly, one has to wait three steps to receive the transmitted message. Of course, the first three bits of the message are lost. In practice, in order to avoid any loss of information, meaningless information which is three samples long is added at the beginning of the message.

Remark 5. In the study of output-memory observability and deadbeat observer design, the results are established for any value of the parameter $\alpha$. On the other hand, the behaviour of the fractional-order Hénon system depends, of course, on this parameter. Indeed, for some values of $\alpha$ the system can converge towards a fixed point, while for other values of $\alpha$ it can become unstable. The most important in the application of the chaotic system in the design of secure communication schemes, which is our goal, is that the system exhibits chaotic behaviour whose strange attractor can be reached for initial conditions judiciously chosen in the basin of attraction.

To illustrate this, we give, in Fig. 4, the bifurcation diagram versus the parameter $\alpha$ for $L=5, a=1.5, b=$ 0.1 . As we can see, the system presents chaotic behaviour for $\alpha$ between 0.76 and 1.1. However, for $\alpha<0.76$ and $\alpha>1.1$, the system becomes unstable. In the case of fractional linear discrete systems, a study of the stability as a function of the memory length $L$ and of the order $\alpha$ has been proposed by Dzieliński and Sierociuk (2008). In the case of non-linear systems, although stability has been the subject of some studies (e.g., Wyrwas et al., 2015), the investigation of stability with respect to the parameters $\alpha$ and $L$ remains, to our knowledge, an open problem. 


\section{Numerical simulations}

The efficiency of the proposed technique of encryption is illustrated by the following simulation results.

6.1. Example 1: Signal reconstruction. In this first example, the message $w(t)$ is generated randomly and is represented in Fig. 5. The amplitude of the signal $w(t)$ is chosen sufficiently small in order to maintain the chaotic behaviour of the fractional-order generalized Hénon map as depicted in Fig. 6, where the chaotic attractor is plotted in the $\left(x_{1}, x_{3}\right)$ plane. As pointed out in the previous section, the secret message $w(t)$ is injected in the dynamics of the third variable $x_{3}(t)$, while the transmitted signal is the second variable $x_{2}(t)=y(t)$. The signal $y(t)$ transmitted via the public channel is reported in Fig. 7, which illustrates the unintelligibility character. The effectiveness of the synchronization between the transmitter and the receiver achieved by the proposed dead-beat observer is illustrated in Figs. 8 and 9 where the true variables $x_{1}$ and $x_{3}$ and the reconstructed variables $\hat{x}_{1}$ and $\hat{x}_{3}$ are plotted. It is shown that the first variable $x_{1}$ is reconstructed exactly after one-unit delay and the third variable $x_{3}$ is reconstructed after two units of delay. Then, the synchronization is exactly successful after two-unit delay. The decrypted message $\hat{w}(t)$ is plotted in Fig. 10. It is clear that the message is recovered exactly after three units of delay corresponding to the dimension of the chaotic system. It is important to note that the message was reconstructed accurately in finite time after only three units of delay. This is an advantage over other methods, where the message is estimated asymptotically and with an estimation error converging to zero as $t$ goes to infinity. This property is interesting in the design of secure speech communication as illustrated in the second example below.

6.2. Example 2: Speech encryption and decryption. The proposed deadbeat observer can be really used for secure speech communication scheme design as basically depicted in Fig. 11. The confidential digitalized speech signal is masked by inclusion in the fractional-order chaotic map. The encrypted drive signal is sent to the receiver throughout the public channel. The proposed deadbeat observer decrypts and recovers the original speech signal.

The following test is performed using MATLAB. The speech signal illustrated in Fig. 12 is sampled at a frequency of $44.1 \mathrm{kHz}$. The encrypted drive signal is illustrated in Fig. 13. The decrypted signal given in Fig. 14 is very similar to the original speech. The proposed speech encryption scheme permits us to recover the original signal very quickly with good performance.

The robustness of a transmission scheme against attacks by intruders depends on the space of the

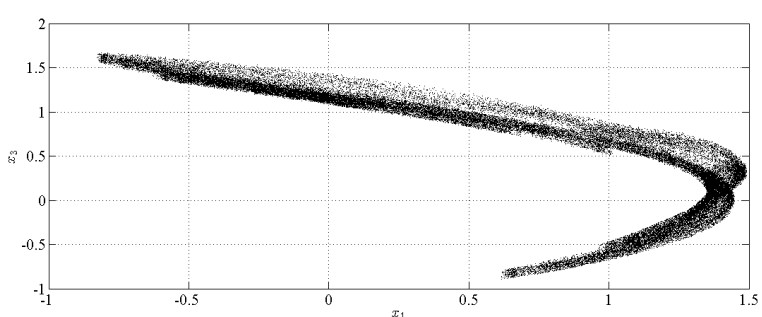

Fig. 6. Strange attractor for the fractional-order generalized discrete Hénon map in the $\left(x_{1}, x_{3}\right)$-plane in the presence of the input $w(t)=0.1 \operatorname{rand}(500,1)$.

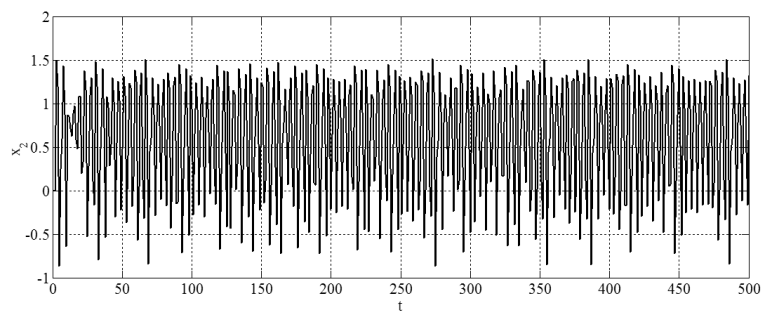

Fig. 7. Signal transmitted $x_{2}$ via the public channel.

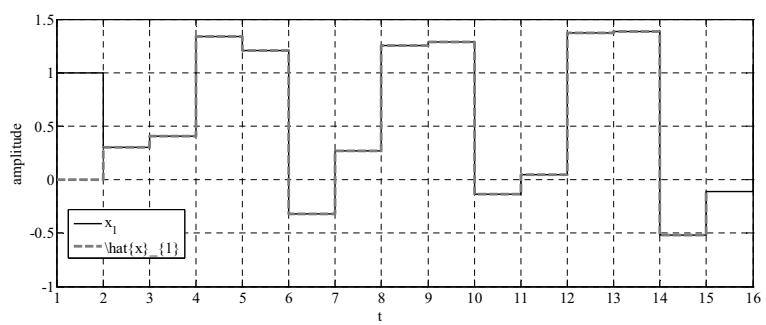

Fig. 8. True state $x_{1}$ (solid line) and reconstructed signal $\hat{x}_{1}$ (dashed line).

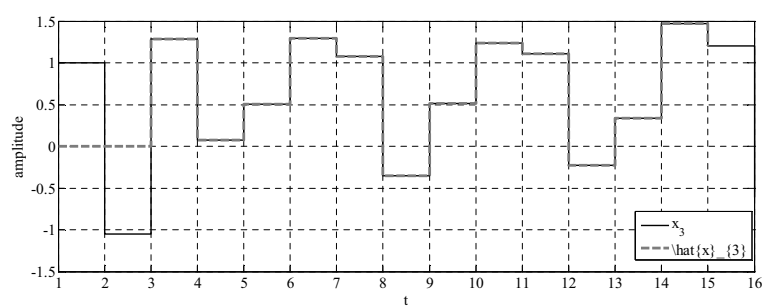

Fig. 9. True state $x_{3}$ (in continuous line) and reconstructed signal $\hat{x}_{3}$ (in dashed line)

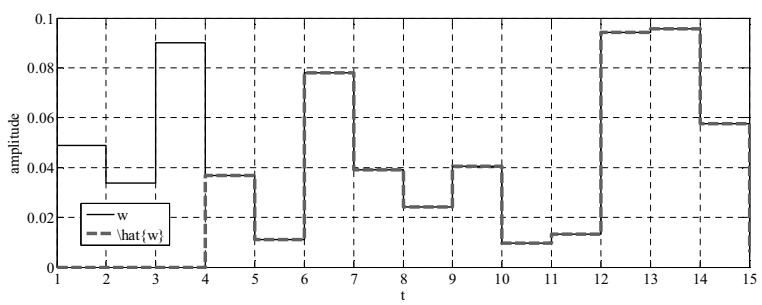

Fig. 10. Original message $w(t)$ (solid line) and reconstructed message $\hat{w}(t)$ (dashed line). 


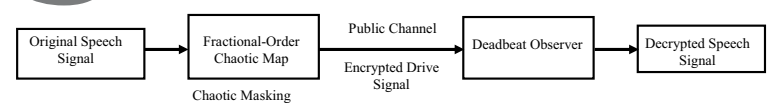

Fig. 11. Block diagram of the speech encryption scheme based on the fractional-order chaotic map.

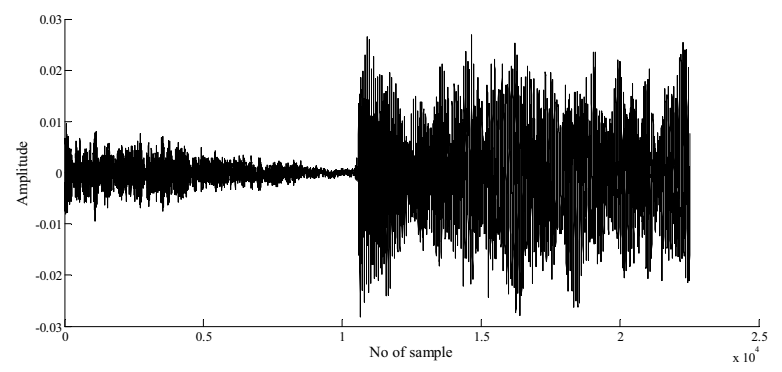

Fig. 12. Histogram of the original speech signal.

security key, which is evaluated by the number of secret parameters constituting this key. The greater the number of these parameters, the higher the transmission scheme. In an integer-order chaotic system based transmission scheme, the parameters of the security key are the parameters of the chaotic system and the initial conditions of the state variables. An intruder who attempts to retrieve secret information must determine these parameters by any means.

The use of fractional order chaotic systems improves the level of security through the fact that the orders of the fractional difference operators are considered additional parameters of the security key. In the case of secure transmission based on integer-order chaotic systems, methods of breaking the key have been proposed, such as as the geometric one (Orue et al., 2008). However, to the best of our knowledge, no method of identifying the orders of fractional difference operators and the length of the memory has been implemented.

In our case, additional parameters constitute the space of the key in addition to the other parameters already used in the integer-order case. These parameters are the fractional orders of the fractional difference operators and the length of the memory. The key space space consists of eight numbers $\left(\alpha_{1}=0.9, \alpha_{2}=0.9, \alpha_{3}=0.9, L_{1}=5\right.$, $L_{2}=5, L_{3}=5, a=1.5, b=0.1$ ), where $\alpha_{i}$ and $L_{i}$ are the order of the fractional difference operator and the length of the memory on the state variable $x_{i}, i=1,2,3$, respectively.

To be able to recover secret information, it is necessary to know the exact values of these parameters. Thus, breaking the security key is a complex, if not impossible, task. To demonstrate this, we study the sensitivity of the proposed secure speech communication scheme to the key parameters. Suppose that an intruder gets in some way approximate values of the parameters of the key. Denote by $\left(\tilde{\alpha}_{1}, \tilde{\alpha}_{2}, \tilde{\alpha}_{3}, \tilde{L}_{1}, \tilde{L}_{2}, \tilde{L}_{3}, \tilde{a}, \tilde{b}\right)$ these approximate values.

In Figs. 16(a), 16(b), 17(a), 17(b), the following intruder's parameters: $\left(\tilde{\alpha}_{1}=0.901, \tilde{\alpha}_{2}=0.9, \tilde{\alpha}_{3}=0.9\right.$, $\left.\tilde{L}_{1}=5, \tilde{L}_{2}=5, \tilde{L}_{3}=5, \tilde{a}=1.5, \tilde{b}=0.1\right),\left(\tilde{\alpha}_{1}=0.899\right.$, $\tilde{\alpha}_{2}=0.9, \tilde{\alpha}_{3}=0.9, \tilde{L}_{1}=5, \tilde{L}_{2}=5, \tilde{L}_{3}=5, \tilde{a}=1.5$, $\tilde{b}=0.1),\left(\tilde{\alpha}_{1}=0.9, \tilde{\alpha}_{2}=0.9, \tilde{\alpha}_{3}=0.9, \tilde{L}_{1}=4, \tilde{L}_{2}=\right.$ $\left.5, \tilde{L}_{3}=5, \tilde{a}=1.5, \tilde{b}=0.1\right)$, and $\left(\tilde{\alpha}_{1}=0.9, \tilde{\alpha}_{2}=0.9\right.$, $\left.\tilde{\alpha}_{3}=0.9, \tilde{L}_{1}=6, \tilde{L}_{2}=5, \tilde{L}_{3}=5, \tilde{a}=1.5, \tilde{b}=0.1\right)$ are considered, respectively. As depicted in these figures, for a slight mismatch with the real values of the key parameters, the original speech message is not recovered. Then the proposed fractional-order chaotic system based communication scheme guarantees high security.

To analyze robustness with respect to the noise of the transmission channel, we assumed that the emitted output sent to the receiver is corrupted by white Gaussian noise and is written as

$$
y_{n}(t)=x_{2}(t)+b(t) .
$$

where $b(t)$ denotes the white Gaussian noise. Figure 17 shows that, for a signal-to-noise ratio of $40 \mathrm{~dB}$, the message could not be reconstructed. Indeed, the observer used is very sensitive to noise because the reconstruction of the states and the secret message is done directly from the noisy output without any filtering process. This is a major drawback of the deadbeat observer. A design of a fractional-order deadbeat Kalman filter would be more appropriate. This is an interesting perspective to consider in future work.

\section{Conclusion}

In this paper, first, some theoretical results on outputmemory observability and the output-memory observability matching condition for nonlinear fractional-order discrete-time systems were introduced. Second, an exact state reconstructor which can be considered a dead beat observer for this class of systems was proposed. Third, an application to the design of a secure speech communication scheme based on a fractional-order chaotic map was developed. The benefit of the new secure data transmission scheme comes from the fact that the secret message is reconstructed accurately and in finite time after a certain number of units of delay equal to the dimension of the chosen chaotic system. As an example, an application to speech encryption was given. However, to evaluate the security performance of the proposed speech encryption system, important features with standard quantitative measure such as statistical analysis, sensitivity analysis, key space analysis, robustness to channel noise and speed performance should be investigated. Future works in these directions are in progress. 


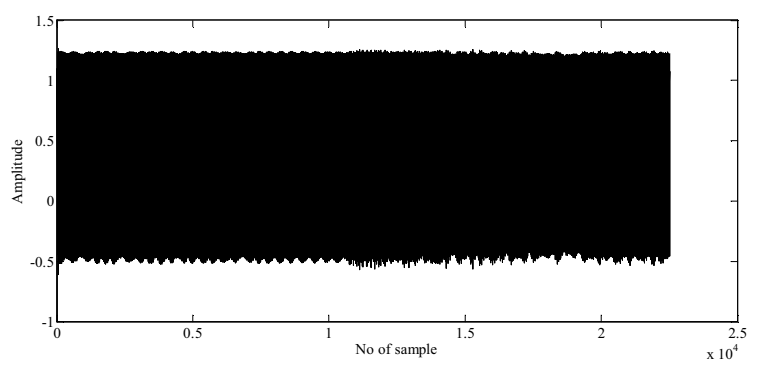

Fig. 13. Histogram of the drive signal.

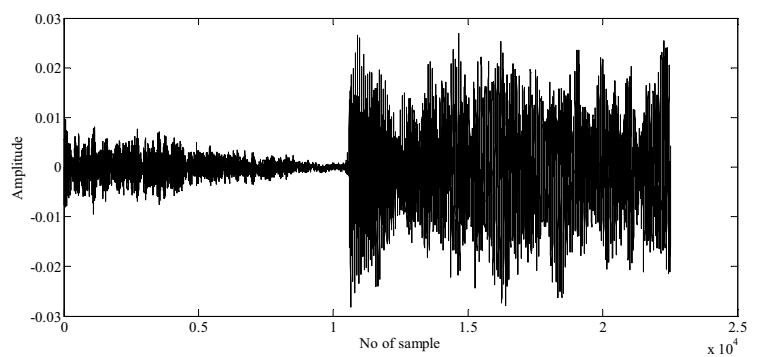

Fig. 14. Histogram of the decrypted speech signal.

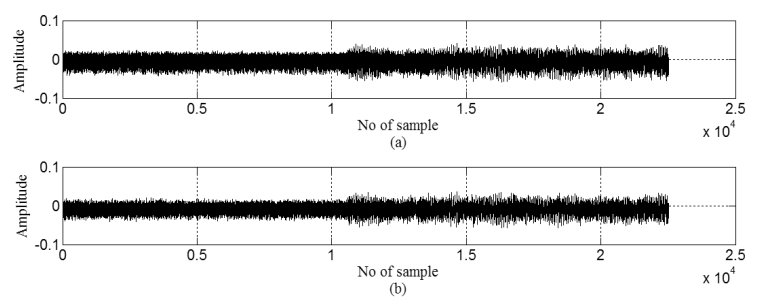

Fig. 15. Key sensitivity analysis: histogram of the decrypted speech signal.
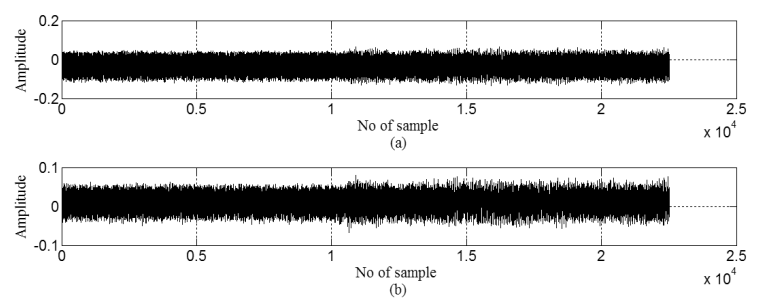

Fig. 16. Key sensitivity analysis: histogram of the decrypted speech signal.

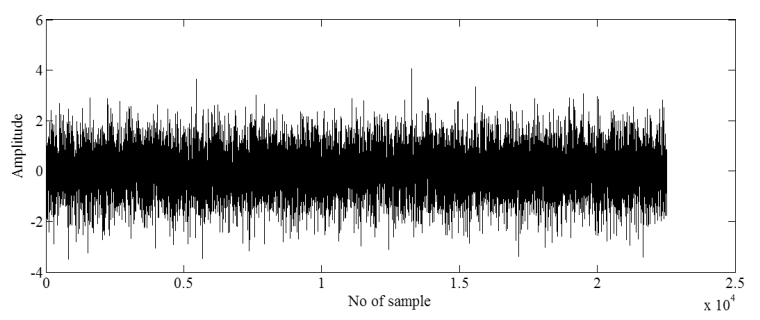

Fig. 17. Histogram of the decrypted speech signal in the presence of a noise signal in the channel.

\section{Acknowledgment}

The authors are grateful to the editor and the three anonymous reviewers for their constructive comments and valuable suggestions, which improved the quality of the paper.

This project was funded by the Centre of Excellence in Intelligent Engineering Systems (CEIES), King Abdulaziz University, under the grant no. CEIES_16_01_02. The authors, therefore, acknowledge the technical and financial support of KAU.

\section{References}

Abdeljawad, T. and Baleanu, D. (2009). Fractional differences and integration by parts, Journal of Computational Analysis and Applications 13(3): 981-989.

Agrawal, S., Srivastava, M. and Das, S. (2012). Synchronization of fractional-order chaotic systems using active control method, Chaos Solitons \& Fractals 45(6): 737-752.

Albertini, F. and D'Alessandro, D. (1996). Remarks on the observability of nonlinear discrete time systems, in J. Doležal and J. Fidler (Eds.), System Modelling and Optimization, Springer, Boston, MA, pp. 155-162.

Albertini, F. and D'Alessandro, D. (2002). Observability and forward-backward observability of discrete-time nonlinear systems, Mathematics of Control, Signals, and Systems 15(4): 275-290.

Atici, F. and Eloe, P.W. (2007). Fractional q-calculus on a time scale, Journal of Nonlinear Mathematical Physics 14(3): 333-344.

Atici, F. and Eloe, P.W. (2009). Initial value problems in discrete fractional calculus, Proceedings of the American Mathematical Society 13(4): 981-989.

Balachandran, K. and Kokila, J. (2012). On the controllability of fractional dynamical systems, International Journal of Applied Mathematics and Computer Science 22(3): 523-531, DOI: $10.2478 / \mathrm{v} 10006-012-0039-0$.

Barbot, J.P., Djemai, M. and Boukhobza, T. (2002). Sliding mode observers, in W. Perruquetti and J.-P. Barbot (Eds.), Sliding-Mode Control in Engineering, CRC Press, New York, NY, pp. 103-130.

Bastos, N.R.O., Ferreira, R.A.C. and Torres, D.F.M. (2011a). Discrete-time fractional variational problems, Signal Processing 91(3): 513-524.

Bastos, N.R.O., Ferreira, R.A.C. and Torres, D.F.M. (2011b). Necessary optimality conditions for fractional difference problems of the calculus of variations, Discrete and Continuous Dynamical Systems 29(2): 417-437.

Belmouhoub, I., Djemai, M. and Barbot, J.-P. (2003). An example of nonlinear discrete-time synchronization of chaotic systems for secure communications, European Control Conference (ECC), Cambridge, UK, pp. 3478-3483. 
Buslowicz, M. (2008). Stability of linear continuous-time fractional order systems with delays of the retarded type, Bulletin of the Polish Academy of Sciences: Technical Science 56(4): 319-324.

Chen, F., Luo, X. and Zhou, Y. (2011). Existence results for nonlinear fractional difference equations, Advances in Difference Equations, Article ID: 713201, DOI: $10.1155 / 2011 / 713201$

Djemai, M., Barbot, P. and Belmouhoub, I. (2009). Discrete time normal form for left invertibility problem, European Journal of Control 15(2): 194-204.

Dzieliński, A. (2016). Optimal control for discrete fractional systems, in A. Babiarz et al. (Eds.), Theory and Applications of Non-integer Order Systems, Lecture Notes in Electrical Engineering, Vol. 407, Springer International Publishing, Cham, pp. 175-185.

Dzieliński, A. and Sierociuk, D. (2008). Stability of discrete fractional state-space systems, Journal of Vibration and Control 14(9-10): 1543-1556.

Eckmann, J.P. and Ruelle, D. (1985). Ergodic theory of chaos and strange attractors, Review of Modern Physics 57(3): 617-656.

Edelman, M. (2018). On the stability of fixed points and chaos in fractional systems, Chaos 28(023112): 023112-1-023112-9.

Feki, M., Robert, B., Gelle, G. and Colas, M. (2003). Secure digital communication using discrete-time chaos synchronization, Chaos, Solitons and Fractals 18(4): 881-890.

Ferreira, R.A.C. and Torres, D.F.M. (2011). Fractional $h$-difference equations arising from the calculus of variations, Applicable Analysis and Discrete Mathematics 5(1): 110-121.

Guermah, S., Djennoune, S. and Bettayeb, M. (2008a). Asymptotic stability and practical stability of linear discrete-time fractional order systems, 3rd IFAC Workshop on Fractional Differentiation and its Applications, Ankara, Turkey.

Guermah, S., Djennoune, S. and Bettayeb, M. (2008b). Controllability and observability of linear discrete-time fractional-order systems, International Journal of Applied Mathematics and Computer Science 18(2): 213-222, DOI: 10.2478/v10006-008-0019-6.

Hanba, S. (1982). Further results on the uniform observability of discrete-time nonlinear systems, IEEE Transactions on Automatic Control 55(4): 1034-1038.

Hénon, M. (1976). A two-dimensional mapping with a strange attractor, Communications in Mathematical Physics 50(1): 69-77.

Holm, M. (2011). The Laplace transform in discrete fractional calculus, Computers \& Mathematics with Applications 62(3): 1591-1601.

Jakubczyk, B. and Sontag, E. (1990). Controllability of nonlinear discrete time systems: A Lie-algebraic approach, SIAM Journal of Control and Optimization 28(1): 1-33.
Kaczorek, T. (2016). Reduced-order fractional descriptor observers for a class of fractional descriptor continuous-time nonlinear systems, International Journal of Applied Mathematics and Computer Science 26(2): 277-283, DOI: 10.1515/amcs-2016-0019.

Khanzadeh, A. and Pourgholi, M. (2016). Robust synchronization of fractional-order chaotic systems at a pre-specified time using sliding mode controller with time-varying switching surfaces, Chaos Solitons \& Fractals 91: 69-77.

Liao, X., Gao, Z. and Huang, H. (2013). Synchronization control of fractional-order discrete-time chaotic systems, European Control Conference (ECC), Zürich, Switzerland, pp. 2214-2219.

Liu, Y. (2014). Discrete chaos in fractional Hénon maps, International Journal of Nonlinear Science 18(3): 170-175.

Luo, C. and Wang, X. (2013). Chaos generated from the fractional-order Chen system and its application to digital secure communication, International Journal of Modern Physics C 24(4): 1350025.

Magin, R.L. (2004). Fractional Calculus in Bioengineering, Begell House Publishers, Danbury, CT.

Miller, K. and Ross, B. (1989). Fractional difference calculus, Proceedings of the International Symposium on Univalent Functions, Fractional Calculus and Their Applications, Koriyama, Japan, pp. 139-152.

Monje, C.A., Chen, Y.Q., Vinagre, B.M., Xue, D.Y. and Feliu, Y. (2010). Fractional-Order Systems and Control: Fundamentals and Applications, Springer-Verlag, London.

Mozyrska, D. and Bartosiewicz, Z. (2010). On observability concepts for nonlinear discrete-time fractional order control systems, in D. Baleanu et al. (Eds.), New Trends in Nanotechnology and Fractional Calculus Applications, Springer International Publishing, Cham, pp. 305-312.

Mozyrska, D., Girejko, E. and Wyrwas, M. (2013a). Comparison of h-difference fractional operators, in W. Mitkowski et al. (Eds.), Advances in the Theory and Applications of noninteger Order Systems, Springer International Publishing, Cham, pp. 191-197.

Mozyrska, D. and Pawłuszewicz, E. (2010). Observability of linear q-difference fractional-order systems with finite initial memory, Bulletin of the Polish Academy of Sciences: Technical Sciences 58(4): 601-605.

Mozyrska, D. and Pawłuszewicz, E. (2011). Linear q-difference fractional order systems with finite memory, Acta Mechanica and Automatica 5(2): 69-73.

Mozyrska, D. and Pawłuszewicz, E. (2012). Fractional discrete-time linear control systems with initialisation, $I n$ ternational Journal of Control 85(2): 213-219.

Mozyrska, D., Pawłuszewicz, E., and Wyrwas, M. (2013b). Observability of h-difference linear control systems with two fractional orders, 14th International Carpathian Control Conference (ICCC-2013), Rytro, Poland, pp. 292-296.

Mozyrska, D., Pawłuszewicz, E. and Wyrwas, M. (2015). The h-difference approach to controllability and observability of fractional linear systems with Caputo-type operator, Asian Journal of Control 17(4): 1163-1173. 
Munkhammar, J. (2013). Chaos in a fractional order logistic map, Fractional Calculus and Applied Analysis 16(3): 511-519.

N'Doye, I., Darouach, M., Voos, H. and Zasadzinski, M. (2016). Design of unknown input fractional-order observers for fractional-order systems, International Journal of Applied Mathematics and Computer Science 23(3): 491-500, DOI: 10.2478/amcs-2013-0037.

Nijmeijer, H. and Mareels, I.M.Y. (1997). An observer looks at synchronization, IEEE Transactions on Circuits and Systems I: Fundamental Theory and Applications 44(10): 882-890.

Nijmeijer, M. (1982). Observability of discrete time nonlinear systems: A geometric approach, International Journal of Control 36(5): 865-871.

Ortigueira, M.D. (2000). Introduction to fractional linear systems. Part 2: Discrete-time case, IEE Proceedings: Vision Image and Signal Processing 14(1): 62-70.

Orue, A.B., Fernandex, V., Alvarez, G., Pastor, G., Romera, M., Li, S. and Montoy, F. (2008). Determination of the parameters for a Lorenz system and application to break the security of two-channel chaotic cryptosystems, Physics Letters A 372(34): 5588-5592.

Pareek, N., Patidar, V. and Sud, K. (2006). Image encryption using chaotic logistic map, Image and Vision Computing 24(9): 926-934.

Pawłuszewicz, E. and Mozyrska, D. (2013). Local controllability of nonlinear discrete-time fractional order systems, Bulletin of the Polish Academy of Sciences: Technical Sciences 61(1): 251-256.

Pecora, L. and Carroll, T. (1990). Synchronization in chaotic systems, Physical Review Letters 64(8): 821-825.

Peng, G.J., Jiang, Y.L. and Chen, F. (2014). Generalized projective synchronization of fractional-order chaotic systems, Physica A: Statistical Mechanics and Its Applications 387(14): 3738-3746.

Petras, I. (2011). Fractional-Order Nonlinear Systems: Modelling, Analysis and Simulation, Springer, Dordrecht.

Podlubny, I. (1998). Fractional Differential Equation, Academic Press, New York, NY.

Podlubny, I. (2003). Geometric and physical interpretation of fractional integral and fractional derivatives, Journal of Fractional Calculus 5(4): 367-386.

Richter, H. (2002). The generalized Hénon maps: Examples for higher dimensional chaos, International Journal of Bifurcation and Chaos 12(6): 1371-1381.

Sabatier, J., Agrawal, O. and Machado, J.T. (2008). Advances in Fractional Calculus: Theoretical Developments and Applications in Physics and Engineering, Springer, Berlin.

Shao, S., Chen, M. and Yan, X. (2016). Adaptive sliding mode synchronization for a class of fractional-order chaotic systems with disturbance, Nonlinear Dynamics 83(4): 1855-1866.
Sharma, V., Agrawal, V., Sharma, B.B. and Nath, R. (2016). Unknown input nonlinear observer design for continuous and discrete time systems with input recovery scheme, Nonlinear Dynamics 85(1): 645-658.

Sira-Ramirez, H., Aguilar-Ibaaez, C. and Suarez-Castaan, M. (2002). Exact state reconstruction in the recovery of messages encrypted by the state of nonlinear discrete-time chaotic systems, International Journal of Bifurcation and Chaos 12(1): 169-177.

Sira-Ramirez, H. and Rouchon, P. (2001). Exact state reconstructors in nonlinear discrete-time systems control, European Union Nonlinear Control Network Workshop, Sheffield, UK.

Tarasov, V.E. (2010). Fractional Zaslavsky and Hénon discrete maps, in C.J. Luo and V. Afraimovich (Eds.), Longrange Interaction, Stochasticity and Fractional Dynamics, Springer, Berlin/Heidelberg, pp. 1-26.

Trujillo, J.J. and Ungureanu, V.M. (2018). Optimal control of discrete-time linear fractional order systems with multiplicative noise, International Journal of Control 91(1): 57-69.

Wolf, A., Swith, J.B., Swinney, H.L. and Vastano, J.A. (1985). Determining Lyapunov exponents from a time series, Physica D: Nonlinear Phenomena 16(3): 285-317.

Wu, G. and Baleanu, D. (2014a). Chaos synchronization of the discrete fractional logistic map, Signal Processing 102: 96-99.

Wu, G. and Baleanu, D. (2014b). Discrete fractional logistic map and its chaos, Nonlinear Dynamics 75(1-2): 283-287.

Wu, G.-C. and Baleanu, D. (2015). Jacobian matrix algorithm for Lyapunov exponents of the discrete fractional maps, Communication in Nonlinear Sciences and Numerical Simulation 22(1-3): 95-100.

Wu, G.-C., Baleanu, D. and Lin, Z.-X. (2016). Image encryption technique based on fractional chaotic time series, Journal of Vibration and Control 22(8): 2092-2099.

Wua, G.C., Baleanu, D., Xie, H.-P. and Chen, F.-L. (2016). Chaos synchronization of fractional chaotic maps based on the stability condition, Physica A: Statistical Mechanics and Its Applications 460: 374-383.

Wyrwas, M., Pawłuszewicz, E. and Girejko, E. (2015). Stability of nonlinear h-difference systems with $n$ fractional orders, Kybernetika 51(1): 112-136.

Xi, H.L., Yu, S.M., Zhang, R.X. and Xu, L. (2014). Adaptive impulsive synchronization for a class of fractional-order chaotic and hyperchaotic systems, Optik, International Journal for Light and Electron Optics 125(9): 2036-2040.

Zhen, W., Xia, H., Ning, L. and Xiao-Na, S. (2012). Image encryption based on a delayed fractional-order chaotic logistic system, Chinese Physics B 21(5): 050506. 


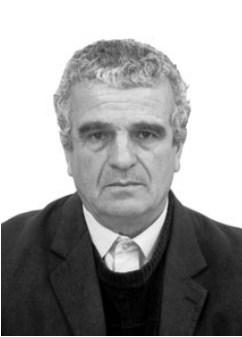

Said Djennoune received the BS, MS, and $\mathrm{PhD}$ degrees in electrical engineering respectively from the National Polytechnic School, Algeria, in 1980, from the High Commission for Research in Algeria in 1986, and from the University of Tizi-Ouzou, Algeria, in $1999 . \mathrm{He}$ worked as a research assistant at the High Commission for Research in Algeria from 1986 to 1988. In 1988, he joined the Department of Automatic Control at Mouloud Mammeri University (Tizi-Ouzou, Algeria). He became a senior lecturer 1999 and a professor in 2005. His research interest is in model order reduction, singular perturbations, nonlinear control, sliding mode control, nonlinear observer design, fractional-order dynamic modeling and control, robust control, hybrid systems, chaotic systems and data secure communication, networked control system, fault diagnosis and fault tolerant control, and vehicle dynamic and control.

Maamar Bettayeb received the BS, MS, and PhD degrees in electrical engineering from the University of Southern California, Los Angeles, in 1976, 1978 and 1981, respectively. He worked as a research scientist at the Bellaire Research Center at Shell Oil Development Company, Houston, USA, in 1981/1982. From 1982 to 1988, he directed the Instrumentation and Control Laboratory of the High Commission for Research in Algeria. In 1988, he joined the Electrical Engineering Department at the King Fahd University of Petroleum and Minerals, Dhahran, Saudi Arabia. He has been a professor at the University of Sharjah, UAE, since 2000. He has published over 300 journal and conference papers in the fields of control and signal processing. He has also supervised over 50 $\mathrm{MSc}$ and $\mathrm{PhD}$ students. His recent research interest is in optimal control, rational approximation, signal and image processing, process control, networked control systems, fractional dynamics and control, nonlinear estimation and filtering, soft computing, wavelets, renewable energies and engineering education.

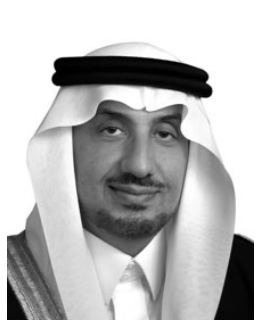

Ubaid M. Al-Saggaf received his BSc degrees (Hons.) in electrical engineering and in mathematics from the King Fahd University of Petroleum and Minerals, Dhahran, Saudi Arabia, in 1980, and his MSc and PhD degrees in electrical engineering from Stanford University, USA, in 1983 and 1986, respectively. He was appointed an assistant professor with the King Fahd University of Petroleum and Minerals in 1986 and then as an associate professor in 1991. He was on leave from 1992 to 2010 and worked for the Ministry of Defense and Aviation as an executive technical advisor for the Research and Development Department. He joined the Electrical and Computer Engineering Department, King Abdulaziz University, in 2010, where he is currently a professor. His fields of interests and specializations cover a wide spectrum, from theoretical to practical aspects of engineering, including systems, control, signal processing, communications, and optronics. He has published several journal and conference papers, and has supervised and co-supervised several MSc and PhD students.

Received: 3 January 2018 Revised: 5 July 2018

Re-revised: 4 October 2018 Accepted: 18 October 2018 